\title{
La gobernanza regional de la cooperación Sur-Sur en América Latina
}

\author{
Paula Ximena Ruiz-Camacho*
}

\begin{abstract}
RESUMEN
La cooperación Sur-Sur se ha consolidado como una modalidad complementaria a la tradicional cooperación Norte-Sur que, en la práctica, se ha materializado como un instrumento para el intercambio de ideas, políticas y experiencias, que se ejecuta entre países en desarrollo que tienen, o creen tener, elementos políticos, históricos y socioeconómicos afines. Sus orígenes se remontan a la década de los cincuenta, y desde entonces, la estructura ideológica que le ha dado forma ha estado influenciada por ideas y hechos que se interrelacionan a partir de distintas narrativas construidas por los estados, subrayando intereses comunes sobre el lugar que ocupan dentro de la arquitectura de la cooperación al desarrollo. A lo largo del siglo XXI, dicha modalidad ha ido ganando mayores espacios de concertación y negociación como un instrumento político de los países en desarrollo que se sustenta en los principios de horizontalidad, solidaridad, reciprocidad e independencia. Desde un enfoque regional,América Latina, por ejemplo, ha favorecido la creación de instrumentos técnicos que, desde la conceptualización hasta el registro de datos, han llevado a la creación de estructuras normativas para la ejecución de la cooperación Sur-Sur. En esa línea argumentativa, desde un enfoque social constructivista de difusión de ideas, normas y prácticas, este artículo analiza de manera descriptiva y explicativa cómo la región ha fortalecido la práctica de la cooperación Sur-Sur a partir de la identificación de problemáticas comunes y discursos encaminados a definirla desde las estructuras ideacionales. El objetivo de este artículo es entender el proceso de formación de la gobernanza regional de la cooperación Sur-Sur en América Latina, para lo cual, se analiza el rol de la Secretaria General lberoamericana desde cuya institucionalidad, los países de la región fortalecen la difusión de prácticas e ideas que podrían significar avances para la construcción de una gobernanza global de la cooperación Sur-Sur.
\end{abstract}

\section{Palabras clave}

Cooperación Sur-Sur; gobernanza regional;América Latina; difusión; constructivismo social.

\section{TITLE}

Regional Governance of the South-South cooperation in Latin America

\section{Abstract}

The South-South cooperation (SSC) dates back to the fifties, and since then it has evolved as a socially constructed idea by developing countries around a collective identity, the main principles on which it has been formed being horizontality, solidarity, reciprocity and independence. The ideological structure that has shaped it has been influenced by ideas and facts that are interrelated from different narratives. Norms and institutions have been the result of transformations in the international development cooperation architecture, especially after the end of the ColdWar.

As a political instrument, the SSC has gained greater scope for consultation and negotiation throughout the 2 Ist century. From the discourse, it has been consolidated as a complement to North-South cooperation, in its practice is an instrument for the exchange of ideas, policies and experiences between developing countries that have, or think they have, related political, historical and socioeconomic characteristics.

Norms, identities and interests are key concepts in the process of the SSC ideas construction. Therefore, the social constructivism of policy diffusion is the approach used for this analysis. It allows for asking questions and understanding world politics and its social structure, as well as the possibility of change in international relations, especially the place of the Southern countries.

\section{DOI:}

http://doi.org//0.15366/relacionesinternacionales2020.44.005

Formato de citación recomendado:

RUIZ-CAMACHO, Paula Ximena (2020), “La gobernanza regional de la cooperación Sur-Sur en América Latina”, Relaciones Internacionales, $n^{\circ} 44$, pp. 87- 105.
* Paula

Ximena RUIZ-

CAMACHO,

Doctoranda en

Estudios Políticos y

Relaciones

Internacionales

(Universidad

Externado de

Colombia y

Universidade de São

Paulo, Brasil). Docente

e investigadora

de la Facultad de

Finanzas, Gobiernoy

Relaciones

Internacionales,

Universidad Externado

de Colombia. Sus

líneas de investigación

incluyen el Sur

global, cooperación

Sur-Sur en América

Latina y cooperación

internacional al

desarrollo.

Recibido:

21/11/2019

Aceptado:

24/05/2020 
The main propose of this article is to understand the process of the regional governance formation process of the SSC in Latin America during the XXI century, analyzing the role of the lberoamerican General Secretariat as an international organization that has allowed the political diffusion of ideas and practices between the countries of the region. As a vehicle for the political diffusion of ideas, practices and experiences among the countries of the region, this organization represents a step forward for the construction of a global SSC.

To analyze it from the SSC ideational structures, the methodology used is a descriptive and interpretive analysis, for which a comprehensive review of the constructivist literatures is made to give shape to the theoretical and conceptual framework on the SSC study. Regarding the historical and conceptual approach to SSC, the most cited academic texts were mainly reviewed, as well as the official sources of the countries indicated in the third part and the annual reports of the lberoamerican General Secretariat.

For constructivist scholars, the agent-structure problem raised by Wendt (1987) continues to be an object of investigative interest and also of division, and its study agenda extends to the understanding of various social phenomena, like the SSC case. In the field of international relations, the SSC has been studied to understand it from two perspectives: on the one hand, from the structure, to understand the changes in the distribution of power within international development cooperation, and on the other hand, from the agent (state), which is the focus of this article, to observe the rise of the emerging powers and their behavior in international politics.

The SSC, beyond being a technical instrument for the exchange of good practices, is also a field of growing academic and research interest, as reflected by the development of the text with the bibliographic sources used for the analysis. Most of the elements that are included around the analysis are part of the constructivist research agenda, such as organizational structures, narratives, ideas, and even the way in which facts and ideas interrelate to shape the institution of the SCC, as well as the formation of identities and the action and behavior of the states.

This article is developed in three sections. The first one, is a theoretical and conceptual review of social constructivism from the perspective of the transnational diffusion of ideas, policies and norms, something that has not been studied enough in the international relations agenda. This is structured as a fundamental guideline for the analytical research. To review the concept of diffusion, first the definition given by Dolowitz and Marsh (2002) of policy transfer is analyzed, a concept that is borrowed from political science, as well as the mechanisms for its diffusion (emulation, coercion, learning and competition), which is subsequently extended to the field of international relations as policy diffusion based on the analysis of Marsh and Sharman (2009)

The second part of the article describes the historical evolution of the SSC from its ideational structures. Its purpose is to understand how ideas and facts influence each other and strengthen relations between states via cooperation. Finally, the third part analyzes the process of the national appropriation of SSC in Latin America based on political diffusion through mechanisms such as emulation and learning.

From this approach, it is understood that the formation of organizational structures has also been a strength of the regional governance process, where the Latin America countries have used the institutionalization created by the lberoAmerican General Secretariat for the systematization of CSS practices. In Latin America, the SSC is consolidated as a tool of regional governance (Ayllón, 2016), because through it, norms have been gradually built that facilitate the exchange of ideas, experiences, public policy development strategies and knowledge that spreads to other geographic regions like a norm cascade (Finnemore, Sikkink, 1998).

Governance is a transversal concept within the analysis, this is understood from the classic definition of the Global Governance Commission (1995) that indicates that it is the sum of many ways in which individuals and institutions, public and private, manage common affairs. For the case that is analyzed on the regional governance of the SSC in Latin America, it is understood as the set of narratives, practices and low principles that integrate different agents around common interests.

Inside the new architecture of development cooperation, the political and analytical notion of governance is fundamental. States are no longer capable of solving problems on their own (Karns, Mingst and Stiles, 2015), and moreover require the cooperation of others and the coordination of interests based on the perception of common problems. This serves to understand the regional governance process in Latin America, the SSC being an instrument for its formulation and management.

Finally, it is concluded that the SSC in Latin America has advanced in the consolidation of technical factors, of how to define and execute it, as same as political factors of how to conceive and guide it based on the construction of a narrative that has been shaped around some common interests and principles. However, the SSC is still a subjective conception of the countries of the South, in which domestic factors determine the dynamism of the diffusion process, as well as their instrumentalization from the political sphere.

\section{KEYWORDS}

South-South cooperation; regional governance; Latin America; diffusion; social constructivism. 


\section{$\mathrm{I}$}

\section{ntroducción}

La cooperación Sur-Sur (CSS) es entendida como una idea socialmente construida por los países en desarrollo alrededor de una identidad colectiva, cuyas normas e instituciones han sido el resultado de las transformaciones de la arquitectura de la cooperación internacional al desarrollo (CID), en especial tras el fin de la Guerra Fría. Uno de sus propósitos es el de promover $y$ fortalecer la capacidad institucional de los gobiernos para el intercambio de ideas, conocimientos y experiencias (Naciones Unidas, 2010).

Normas, identidades e intereses son conceptos claves dentro del proceso de formación de las ideas que moldean la CSS en la práctica, por lo cual, este artículo se aborda desde el enfoque social constructivista. Los factores ideológicos (ideas, identidades, roles) y de liderazgo (intereses, instituciones, normas) de los agentes, en este caso estatales (Milani, 20l8), son fundamentales para comprender la CSS dentro de las Relaciones Internacionales (Jiménez, 2003;Ayllón, 2007; Pauselli, 2013; Ojeda, 2016; Santander, 2016).

En cuanto a su definición, la CSS es polisémica y la manera de entenderla o de concebirla es intersubjetiva, los agentes (estados) la perciben, definen y ejecutan de maneras diferentes por lo cual, se encuentran diversas definiciones. Dentro de este amplio marco conceptual, independientemente de si su origen es estatal, académico o si proviene de organismos multilaterales, regionales o foros ad hoc, se evidencian dos elementos dentro del análisis: por un lado, su carácter político, al tratar de darle un espacio autónomo dentro de la arquitectura de la cooperación y, por el otro, el social que se articula con las agendas globales para el desarrollo.

En términos generales, la CSS es una "construcción social moldeada por la relación dialéctica entre conocimiento y poder" (Bergamaschi y Tickner, 2017, p. 10) por lo que dentro de este análisis resulta fundamental abordar el fenómeno de la CSS a partir de las estructuras ideacionales que la sustentan. Para comprender el comportamiento de los actores y la consolidación de sus intereses, los constructivistas sociales incorporan en su agenda investigativa "el estudio de la influencia que las ideas ejercen sobre las acciones. La causalidad ideacional” (Ibáñez, 20I5, p. 197), que en este caso son los principios a través de los cuales se ha construido la CSS:"el respeto de la soberanía nacional, la implicación nacional y la independencia, la igualdad, la no condicionalidad, la no injerencia en los asuntos internos y el beneficio mutuo" (Naciones Unidas, 20l0, p. 4).

En el 2019 se conmemoraron los cuarenta años de la ejecución de la cooperación técnica entre países en desarrollo (CTPD), una iniciativa impulsada desde 1978 por el Programa de Naciones Unidas para el Desarrollo (PNUD), para dinamizar el intercambio de conocimientos, experiencias y prácticas entre estados con problemáticas socioeconómicas afines (UNDP, 1978).

Conceptualmente la CTPD evolucionó a lo que hoy se denomina CSS, las narrativas, prácticas y principios bajo los que esta modalidad se ha ido consolidando, evidencian el interés de los países en desarrollo por construir consensos a través de diversos espacios de gobernanza, es decir, a partir de "la suma de muchas formas en que individuos e instituciones, públicas y privadas, manejan asuntos en común" (Commission on Global Governance, 1995, p. 16).

Esto último, resulta clave dentro de la nueva arquitectura de la CID porque los estados ya 
no están en la capacidad de resolver los problemas por sí solos (Karns, Mingst y Stiles, 20I5), sino que requieren de la cooperación de unos y otros, del mayor involucramiento de otros actores no estatales para, de esta forma, lograr la "coordinación de intereses a partir de la percepción de problemas comunes" (Ayllón, 2007, p. 33). Para el caso de América Latina, dicha coordinación se ha adelantado principalmente a través de procesos de integración regional, siendo la CSS un eje central de su gestión (Ayllón, Ojeda y Surasky, 20I4; Viola, 20I4).

Por tanto, desde un marco social constructivista de difusión de políticas, este artículo tiene el objetivo de entender el proceso de formación de la gobernanza regional de la CSS en América Latina analizando la consolidación de estructuras organizacionales en algunos países de la región. Posteriormente, se revisa cómo desde la institucionalidad creada por la Secretaría General lberoamericana (SEGIB) se ha favorecido el proceso de difusión de políticas, prácticas e ideas entre los países latinoamericanos que, en un primer momento, favorece la construcción de una gobernanza global de la CSS.

Este análisis se aborda desde tres apartados, en el primero, se hace una revisión teórica y conceptual a partir del constructivismo social utilizando el enfoque de difusión trasnacional de ideas, políticas y normas como pauta analítica. El segundo epígrafe, describe la evolución histórica de la CSS desde sus estructuras ideacionales, es decir, a partir de entender cómo las ideas y los hechos se han influenciado mutuamente para fortalecer las relaciones entre estados vía cooperación. La tercera parte, es más extensa que las anteriores, por cuanto analiza el proceso de apropiación nacional de la CSS en América Latina a partir de la difusión de normas vía emulación y aprendizaje.

A partir de este enfoque, se busca comprender la formación de estructuras organizacionales que, a su vez, han fortalecido el proceso de gobernanza regional, destacándose principalmente el trabajo que se adelanta desde la SEGIB para la sistematización de prácticas de CSS, que ha servido como "una herramienta de gobernanza regional” (Ayllón, 20I6, p. 4I), a través de la cual se han construido paulatinamente normas que facilitan el intercambio de ideas, experiencias, políticas públicas, estrategias de desarrollo y conocimiento que son difundidas a otras regiones geográficas. Siguiendo esta misma línea argumentativa, a pesar de la coyuntura política de cada país, o de sus diferencias socioeconómicas, la CSS en la región ha alcanzado niveles de concertación en diversos escenarios de integración, por lo cual representa "una opción por potenciar la proyección de América Latina y su vinculación con otros países y bloques en el Sur Global” (Ayllón, 2016, p. 42).

\section{Difusión de políticas de CSS a la luz del constructivismo social}

El constructivismo social permite hacer preguntas sobre la política mundial y su estructura. Desde este enfoque se hace énfasis en la dimensión social, en la posibilidad de cambio en las relaciones internacionales, ocupándose de cómo entender el proceso de mutua constitución entre agente y estructura.

No obstante, tal como señalan Finnemore y Sikkink (200I), este último postulado ha sido fuente de división teórica entre académicos constructivistas, siendo el problema agente-estructura, planteado por Wendt (1987, 1992, 1995), uno de los temas alrededor de los cuales ha girado gran parte del debate e investigación académica constructivista (Finnemore y Sikkink, 200 I; Burchill, 
2005; Wight, 2006; Checkel, 2008; Ibáñez, 20I5). Desde esta perspectiva, si bien "las propiedades de los agentes y de las estructuras sociales son relevantes para explicar el comportamiento social" (Wendt, 1987, p. 338) el problema radica en entender cómo agente-estructura se interrelacionan.

Por ejemplo, en el estudio de las Relaciones Internacionales, la CSS ha sido estudiada desde la noción de estructura con el objeto de comprender los cambios en la distribución del poder dentro de la cooperación internacional al desarrollo (Agnew, I998; Mawdsley, 20I 5, 2018).Asimismo, se ha abordado a partir del enfoque de agente (estado) para observar el auge de las potencias emergentes y su comportamiento en la política internacional (Bergamashi y Soulé-Kohndou, 20 I6; Engel y Keijzer, 20I3; Quadir, 20I3; Souza, 20I3). Este último es el enfoque alrededor del cual se aborda este artículo.

Lo anterior evidencia que la CSS, más allá de ser un instrumento técnico para el intercambio de buenas prácticas, es también un fenómeno de creciente interés académico. Su construcción social, la forma como hechos e ideas se interrelacionan para darle forma a su institución ', la formación de identidades y la acción de los estados, entre otros factores que constituyen la CSS, son parte de la agenda investigativa constructivista, la cual ha venido ampliando sus temas y métodos de investigación.

Por tanto, se parte del supuesto de que no hay una única concepción que permita entender cómo es el mundo o cómo debería ser (Wight, 2006), por el contrario, se observa de formas distintas; así sucede, por ejemplo, con los países en desarrollo en la manera como cada uno concibe e instrumentaliza la CSS. Así, la pauta de análisis propuesta para este artículo parte de un enfoque constructivista de difusión que se centra en las normas y se enfoca en los agentes estatales con el propósito de comprender la formación de la CSS en un plano regional y su posible difusión hacia lo global.

Desde una perspectiva sociológica interpretativa, el constructivismo social favorece la comprensión de distintos fenómenos y su proceso ${ }^{2}$ de formación (Wendt, 1992). Para Finnemore y Sikkink (200I) los estudios constructivistas giran alrededor de entender cómo los factores sociales cambian e influencian políticas, e incluso cambios en las reglas ${ }^{3}$.

Esto último, por ejemplo, es abordado en el análisis descriptivo del segundo apartado, en el cual se interpreta la reemergencia de la CSS a inicios del siglo XXI a partir del auge de potencias emergentes del Sur, cuya actuación transforma prácticas en la estructura, e influencia el comportamiento de otros agentes. En este sentido, la construcción de una identidad colectiva alrededor de factores ideacionales le ha dado forma a la realidad social de la CSS y le ha dado subjetividad a su narrativa.

En cuanto al concepto de narrativa, siguiendo a Fraundorfer (20I5), este puede ser abord-

\footnotetext{
"Una colección relativamente estable de prácticas y reglas que definen el comportamiento apropiado para grupos específicos de actores en situaciones específicas. Dichas prácticas y reglas están integradas en estructuras de significado y esquemas de interpretación que explican y legitiman identidades particulares, prácticas y reglas asociadas con ellas” (March y Olsen, 1998, p. 948).

2 Definido por Wendt (1992) como una forma de interacción y de aprendizaje.

3 Una regla es "un mandato que le dice a la gente qué hacer y todas las formas como las personas lidian con las reglas se llaman prácticas. Las reglas les dan opciones a los agentes” (Onuf, 1998, p. 59).
} 
ado como una noción del ejercicio del poder que se manifiesta como forma particular del discurso, como parte de la realidad política de los agentes. Las historias son reinterpretadas para proyectar una visión y mostrar puntos de vista que se transmiten a través de un discurso que, a su vez, es una herramienta utilizada por los actores políticos para influenciar y cambiar realidades o, en última instancia, darles sentido a sus decisiones.

A partir de lo anterior, la estructura ideacional que ha moldeado la CSS se evidencia en una narrativa cargada de símbolos, que evidencia creencias sobre el deber ser del intercambio entre iguales, significados sobre el lugar y el rol que desempeñan en materia de CID, y conocimientos colectivos alrededor de la práctica que se sustenta en los principios que la rigen.

A partir del enfoque de difusión de políticas, lo que se observa en el caso de América Latina, es que la CSS se ha dinamizado a partir de los agentes estatales, los cuales impulsan el proceso de difusión, diseñan las normas y favorecen o entorpecen su acción. A partir de este postulado, se entiende que son los agentes los que dan significado a la realidad, su autonomía es intersubjetiva y el conjunto de sus interacciones ayuda a construir, reproducir y transformar las estructuras (Burchill, 2005).

El estado como agente se entiende a partir de las estructuras organizacionales (internas) que le han dado forma a las identidades e intereses de los actores (Wendt, 1995). En este sentido, la estructura está impregnada de factores ideacionales que definen los intereses de los agentes (Ruggie, 1998). Dentro de las estructuras organizacionales, las decisiones individuales son implementadas al interior del aparato estatal, desde donde se rigen prácticas y normas para la implementación de políticas que, una vez adoptadas y en caso de ser exitosas o satisfactorias, se difunden.

La idea alrededor de la transferencia de políticas ha sido abordada principalmente por la Ciencia Política, destacándose las investigaciones de Dolowitz y Marsh (2002), alrededor del concepto de policy transfer, "conocimiento acerca de las políticas, arreglos administrativos, instituciones e ideas en un sistema político (pasado o presente) es usado en el desarrollo de políticas, arreglos administrativos, instituciones e ideas en otro sistema" (p. 5).

Marsh y Sharman (2009) van más allá y lo amplían al campo de las Relaciones Internacionales, donde se privilegia el análisis en la estructura, y no tanto en la agencia como se ha tendido a hacer en la Ciencia Política. Por tanto, dentro del campo de las Relaciones Internacionales se hace énfasis en la "difusión de políticas", un concepto que se ha incorporado en el constructivismo social, cuya agenda ha tendido a ampliarse, y centran su atención en los problemas de la complejidad de la norma y en cómo se estructura (Fehl, 2019).

La difusión de normas, ideas, prácticas y políticas se da debido a la creciente interdependencia entre los actores (Gilardi, 2013). Dicha difusión puede darse a partir de cuatro mecanismos: emulación, aprendizaje, competencia o coerción, pero dado que el proceso de formación de la CSS latinoamericana se ha desarrollado vía emulación y aprendizaje, se definen estos dos mecanismos que se retoman en el tercer apartado. 
En cuanto al proceso de difusión vía emulación, siguiendo la línea de Gilardi, significa que las características normativas y socialmente construidas de las políticas son más importantes que sus consecuencias objetivas, es decir, "las organizaciones se ajustan a lo que se define socialmente como apropiado y eficiente, sin tener en cuenta el impacto real en el desempeño de la organización" (20I3, p. 466). Un mecanismo descrito por Marsh y Sharman (2009) así:"proceso de copiar modelos extranjeros en términos de factores simbólicos o normativos, en lugar de una preocupación técnica o racional con la eficiencia funcional” (p. 272).

La emulación también puede entenderse a partir de la difusión de normas; en ese sentido, siguiendo la definición de Sikkink (I99I), una norma es un conjunto de reglas y prácticas que rigen unas organizaciones y determinan el comportamiento de los actores dentro de una determinada organización. De manera complementaria, para Finnemore y Sikkink (1998), las normas se envuelven en estándares de comportamiento entre lo "apropiado" y lo "propio" y tienen una dimensión tanto intersubjetiva como evaluativa, esta última vinculada a una cuestión moral sobre el comportamiento de los agentes.

Las autoras analizan el proceso que surte una norma, desde el momento en que se crea por necesidad, "la emergencia de la norma", seguido por un efecto en cascada que se refiere al momento en que la norma se socializa y empieza a darse la difusión y, por último, la internacionalización de la norma, cuando es aceptada y cuya implementación o presencia ya no permite otro tipo de comportamiento. Para el caso de la CSS, las normas son más de tipo constitutivo que regulativo, que se van adaptando conforme a los cambios en las narrativas y los acuerdos políticos. Dichas normas pueden ser regulativas, que ordenan y limitan el comportamiento, o constitutivas, crean nuevos actores, intereses y categorías de acción (Finnemore y Sikkink, 1998).

En cuanto al aprendizaje, hace referencia a como la experiencia de otros puede proporcionar información útil sobre las posibles consecuencias de una política. Para Gilardi, es un "proceso mediante el cual los responsables de las políticas utilizan la experiencia de otros países” (2013, p. 463). Este, por ejemplo, es el mecanismo más practicado por los países latinoamericanos que han desarrollado un activo rol de oferentes de cooperación y difunden sus políticas públicas exitosas, es el caso de Argentina, Brasil, Chile, Colombia, Cuba y México, para destacar a manera de ejemplo los países que han implementado el mayor número de prácticas de CSS como oferentes (SEGIB, 2018).

La CSS ha estado influenciada por las ideas, normas y políticas propias del contexto internacional en el que los países en desarrollo han construido una identidad colectiva. Las ideas que forman la CSS han sido promovidas a nivel global por algunos órganos al interior de las Naciones Unidas, pero muy especialmente desde los organismos regionales, subregionales e interregionales ${ }^{4}$.

Finalmente, dos son las conclusiones que de manera exploratoria se abstraen de lo discutido en este apartado, $y$ hacen referencia a la importancia dentro del análisis constructivista del rol

\footnotetext{
América Latina es la región con el mayor número de foros o escenarios de integración en los que la definición e implementación de la CSS ocupan un espacio importante. En cuanto a lo interregional, se destacan las cumbres América del Sur - África; América del Sur - Asia; Foro de Cooperación de América Latina y Asia del Este (Focalae) y la Cumbre Afroasiática del estado y de gobierno (Bandung + 60). Estos foros han logrado "vincular experiencias históricas y concepciones de la CSS y la cooperación triangular plurales pero unidas por el común denominador de las narrativas y principios (...) que vinculan a los países en desarrollo" (Ayllón, 2018, p. 22).
} 
que tienen las normas y los agentes para comprender el comportamiento de la CSS en la región latinoamericana $y$, de esta forma, el proceso de difusión de políticas.

En primer lugar, el proceso de difusión de políticas, prácticas e ideas se ha dado vía aprendizaje y emulación, factores claves para la consolidación de su institucionalidad. En segundo lugar, que la CSS sigue siendo una práctica principalmente desarrollada por los estados, por lo que se aborda su análisis a partir de su agencia, de su capacidad para llevar a cabo la difusión de un país a otro (Marsh y Sharman, 2009).

\section{Surgimiento de la CSS desde las estructuras ideacionales}

La construcción de la CSS ha estado fundamentalmente ligada a su componente narrativo, por lo que este segundo apartado analiza los hechos sociales y el rol de las ideas en el proceso de formación de esta modalidad. La CSS se ha cimentado dentro de una estructura ideacional en la que se evidencian creencias, significados y conocimientos que, en la práctica, se materializan a través del discurso de los gobiernos que la practican.

Su historia se remonta a la década de los cincuenta con la Conferencia Afroasiática de Bandung (Indonesia), en la que fueron aprobados los diez principios para el establecimiento de las relaciones entre países en desarrollo, que dan sustento a la CSS. Este encuentro les permitió a sus participantes "superar sus diferencias y avanzar en la cooperación política entre sociedades con características similares" (Ayllón, 2013, p. 24).Asimismo, sentó las bases para que una década después se creara el Movimiento de los No Alineados, al que le sucedieron diversos encuentros con el propósito de fortalecer los espacios de diálogo y concertación entre un Sur geopolítico.

Para Ojeda (2016), la década de los setenta fue el escenario propicio para la "búsqueda de la concertación política y la institucionalización" (p. 67) de las alianzas Sur-Sur. Un período en el cual se fortalecieron los ideales políticos de los países en desarrollo alrededor de la cooperación como un instrumento para el desarrollo, e incluso una vía para transformar el imperante orden económico internacional (Milani, 2018, p. 43).

Frente a esto último, a través de las Naciones Unidas los países del Sur buscaban legitimar su acción colectiva. Para 1974 se aprobaron dos resoluciones que pretendían instaurar un nuevo orden económico internacional (Chaturvedi, Fues y Sidiropoulos, 20 I2; Surasky y Ayllón, 2018) y que respondían principalmente a los intereses y a la voluntad de estos por ampliar su participación en los procesos de toma de decisión y negociación a nivel mundial.

Para promover la CTPD, las Naciones Unidas ejercieron un rol fundamental para la difusión de ideas, prácticas y conocimientos, en especial porque era el organismo multilateral en el que los países en desarrollo tenían mayor voz y representatividad. Mediante la Resolución 325 I (XXIX) de 1974, se creó la Unidad Especial del Programa de Naciones Unidas para el Desarrollo, a la cual se le dio el mandato de facilitar la comunicación, difundir el conocimiento, las experiencias y las políticas entre países en desarrollo (Naciones Unidas, 1974). En esa línea, para 1978 esta unidad especial organizó en la capital argentina el primer encuentro sobre CTPD, un hecho histórico que continúa siendo icónico en la formación y definición de la CSS. 
En dicho encuentro, se adoptó el Plan de Acción de Buenos Aires (PABA), que más que un documento político se consolidó como un instrumento técnico para el manejo de la CTPD. En su párrafo introductorio, el PABA destaca que con su aprobación se sientan las bases conceptuales y técnicas para el fortalecimiento de capacidades nacionales (UNDP, 1978).

No obstante, la aprobación del PABA se enmarcó en el contexto de Guerra Fría, en el cual primaban las lógicas ideológicas y políticas que limitaban la capacidad de acción de los estados del Sur. Fue un período de contrastes; por un lado, fue la "gestación fallida de un nuevo orden, de perfiles no claramente definidos" (Surasky y Ayllón, 20I8, p. 4I), que se evidenció en el PABA ante la ausencia de elementos políticos "que hoy son ineludibles: democracia, participación, rendición de cuentas y buen gobierno" (Surasky, 20I5, p. 3). Por otra parte, fue una época en la cual se fortaleció la idea de una identidad del Sur, siendo la CTPD un instrumento para su acción.

Con el fin de la Guerra Fría, la década de los noventa se caracterizó por el auge de cumbres sobre diversas problemáticas que fueron impulsadas desde la ONU (Sanahuja y Tezanos, 2016; Chaturvedi et al., 2012) y "la globalización se convierte en un factor catalizador para el multilateralismo" (Antonini y Hirst, 2009, p. 3 I). Estos encuentros se materializaron en una agenda global para el desarrollo, la denominada Agenda del Milenio (2000) que recoge ocho objetivos, a través de los cuales también se pretendía revitalizar el sistema de ayuda al desarrollo a través del diseño de diversos mecanismos, instrumentos y estrategias para hacerlo más eficaz (Hulme, 2009).

Tras la aprobación de los objetivos de desarrollo del milenio (ODM) y posteriormente, el Consenso de Monterrey (2002) sobre la financiación para el desarrollo, se marcó la pauta frente a la necesidad de encontrar diversos mecanismos políticos y económicos para financiar y fortalecer las estrategias de cooperación con miras a alcanzar metas comunes a nivel global y de esta forma disminuir la pobreza extrema y el hambre en un período de quince años.

Si bien ninguno de los ODM menciona la CSS, el último objetivo señala la necesidad de "fomentar una asociación global para el desarrollo" (Naciones Unidas, 2000) que contempla diversos mecanismos de cooperación entre los que se vinculan actores tanto estatales como no estatales, ampliando la cooperación hacia verdaderos marcos de gobernanza global.

En 2004, la Resolución 58/220 de 2004 de la Asamblea General aprobó que la Unidad Especial para la Cooperación Técnica entre Países en Desarrollo pasara a denominarse Unidad Especial para la Cooperación Sur-Sur (UNOSSC, 20I8), dando así un reconocimiento a los cambios propios en las narrativas de un amplio grupo de países que se perciben como socios para el desarrollo. A este contexto institucional, se le suma el auge de potencias emergentes del Sur, cuya participación en ámbitos internacionales de toma de decisión fortalece una idea del Sur (Mawdsley, 2012; Quadir, 20I3).

Estas nuevas potencias regionales emergentes (Brasil, China, India y Sur África) desempeñan un rol fundamental en el diseño ideológico y normativo de la CSS durante la primera década del siglo XXI. En cuanto a esto último, no solo crean nuevos espacios de integración interregional como el Foro Trilateral India, Brasil y Sur África, sino que participan, de manera activa, a través de la United Nations Office for South South Cooperation (UNOSSC) e incrementan sus 
aportes económicos para fortalecer la financiación de proyectos e investigaciones en materia de CSS y con esto tener un mayor juego político en la definición de estrategias y proyectos dirigidos desde esta oficina (Bergamashi y Soulé-Kohndou, 2016).

El interés por fortalecer mecanismos financieros y económicos va acompañado de la definición y difusión de principios para caracterizar y ejecutar la CSS. La reemergencia de la CSS ha estado cargada de significados en su narrativa que reflejan cambios en las estructuras ideacionales, que se desarrollan fuera de la estructura social construida durante casi cincuenta años por el Comité de Ayuda al Desarrollo (CAD) de la OCDE. Desde el discurso, se fortalece la idea de una cooperación horizontal entre socios, que es dinámica, solidaria y desinteresada, que incorpora un factor moral dentro de su discurso.

Sin embargo, la práctica de la CSS no necesariamente se enfoca en resolver los problemas de desarrollo, sino que se materializa a través de la implementación de diversas estrategias que incorporan también lo comercial, lo político, e incluso de seguridad ${ }^{5}$. Tanto el alcance como la definición de los intereses políticos de la CSS, no dejan de ser una concepción subjetiva de los países del Sur que buscan unificar criterios a través de la consolidación de factores de tipo técnico de cómo definirla y ejecutarla, e incluso de factores políticos de cómo concebirla y guiarla a partir de la construcción de la identidad en una lógica discursiva. Para Mawdsley, la CSS se enmarca en "un régimen simbólico ampliamente compartido creado a través de estrategias discursivas y expresiones particulares" (20I2, p. 268).

América Latina no ha sido una región ajena a este proceso. Desde marcos de gobernanza regional, la narrativa de la CSS se ha instrumentalizado para fortalecer los procesos de integración, dándole forma a la estructura ideacional bajo la que se da la difusión de prácticas, ideas y políticas entre países socios a través de mecanismos como el aprendizaje. No obstante, esta estructura ideacional se muestra compleja, pues a pesar de existir una narrativa colectiva que le ha dado forma, cada país ha surtido su propio proceso de acomodación y reinserción dentro de la institucionalidad de la CSS, tal como se analiza en el siguiente apartado.

\section{La agencia de América Latina y su rol en la ejecución de la CSS}

Como fenómeno de estudio, la CSS se ha transformado conforme a las dinámicas del sistema internacional. Cada momento de auge, estancamiento o retroceso responde a esos cambios que hacen de esta una estructura intersubjetiva y moldeada de ideas, valores y símbolos. Por lo cual, el análisis que acompaña este apartado se centra en un marco temporal que va hasta la publicación del informe de la SEGIB, Una década de Cooperación Sur-Sur en Iberoamérica (2018).

Esta tercera parte, tiene como propósito estudiar el proceso de formación de la CSS en América Latina. En primer lugar, es preciso señalar que la difusión de políticas, prácticas e ideas que se desarrollan en materia de CID son el resultado de un proceso de transferencia llevado a cabo de Norte a Sur, y que se ha dinamizado en las dos últimas décadas de Sur a Sur. Frente a esto último, se analizan las estructuras institucionales de algunos países de la región, los cuales, según cifras y datos suministrados por la SEGIB (2018), son los más dinámicos en el ejercicio de la cooperación en su rol de oferentes.

\footnotetext{
5 Colombia, por ejemplo, es el caso más representativo de cómo se instrumentaliza la CSS en materia de seguridad (Ruiz, 2018).
} 
A partir del concepto de difusión, abordado en el marco teórico y conceptual, se explica la forma en que en un inicio se estructuraron las agencias de CID de algunos países de América Latina, un proceso de difusión vía emulación de normas que se adaptan a las estructuras burocráticas de cada estado (Sikkink, 1991).Asimismo, este concepto de difusión se revisa para observar cómo se da la transferencia vía aprendizaje en la implementación y adecuación de marcos institucionales para la definición y gestión de la CSS en la región.

En cuanto a la difusión política por emulación, Finnemore y Sikkink (1998) señalan la importancia de las normas dentro de las transformaciones políticas que, para el caso de América Latina, son el resultado de la forma en que las políticas y prácticas en materia de cooperación han viajado de Norte a Sur emulando estructuras organizacionales para su gestión. Milani (2018), por ejemplo, identifica a partir de las estructuras organizacionales de las agencias de cooperación de los miembros del CAD, cuatro modelos, que en el caso de América Latina se replican.

En el primer modelo, la responsabilidad sobre el diseño, formulación y ejecución de la CID recae directamente en el ministerio de relaciones exteriores, desde donde se definen las estrategias, los montos, los socios y la política, entre otros. En el segundo modelo, existe una agencia de cooperación que está adscrita a la cancillería desde donde se da línea y se define la estrategia política, pero en cuya agencia recae la responsabilidad de liderar la implementación y puesta en marcha de la agenda de CID.

En el tercer modelo, el ministerio de relaciones exteriores es el encargado de dar una línea política global, y existe una agencia en la que recae cierto grado de autonomía, es decir, que tiene la facultad de ejecutar la CID. Por último, se encuentra el cuarto modelo, en el cual el país crea un ministerio propio o agencia que, junto a la cancillería, definen las líneas políticas, objetivos, montos, estrategias, etc., en materia de $\mathrm{CID}^{6}$.

Tomando los cuatro modelos antes descritos y extrapolándolos al caso de América Latina, se evidencian estructuras institucionales similares entre las que se pueden mencionar algunos ejemplos ${ }^{7}$. Las agencias de Argentina y México emulan la estructura propuesta para explicar el primer modelo, que señala que los temas de CID son manejados desde el ministerio de relaciones exteriores.

En cuanto al segundo, la estructura más representativa es la de Brasil, que incluso para Milani (2018) es un modelo híbrido entre el segundo y el tercer modelo, porque cuenta con una agencia autónoma y está adscrita al ministerio de relaciones exteriores que da línea política; dentro de este modelo se destaca la agencia chilena de cooperación internacional.

Finalmente, está el modelo que adopta una estructura independiente pero complementaria al ministerio de relaciones exteriores en los que la directriz y ejecución en materia de coope-

6 Dentro del análisis descriptivo de los modelos de agencia existentes en los países miembros de la OCDE analizados por Milani están: primer modelo Dinamarca y Noruega; segundo modelo Países Bajos, Finlandia, Grecia, Irlanda, Italia y Suiza; tercer modelo Alemania, Austria, España, Estados Unidos, Francia, Japón, Portugal y Suecia, y cuarto modelo Australia, Canadá y Reino Unido (Milani, 2018, pp. 204-205).

7 Para este ejercicio se analizó la información oficial publicada en las páginas web de cada una de las agencias de cooperación incluidas en el cuadro. 
ración recae sobre algún ministerio, que es el caso de Cuba. En cuanto a la Agencia Presidencial de Cooperación Internacional de Colombia (APC-Colombia) es un modelo complejo, puesto que su agencia está adscrita directamente a la presidencia, trabajan de la mano con el ministerio de relaciones exteriores, e incluso, con otras entidades estatales. Cuenta con autonomía limitada en la toma de decisiones por lo que se clasifica entre un modelo tres y cuatro.

En términos generales, la anterior clasificación muestra cómo se emulan modelos de gestión y de toma de decisión organizacionales desde los miembros del CAD hacia América Latina, una estructura que continúa respondiendo a las dinámicas de la arquitectura de la ayuda al desarrollo. No obstante, a lo largo de este artículo, también se ha analizado cómo los hechos y las ideas se han interrelacionado desde Bandung (1955) hasta PABA+40 (2019) ${ }^{8}$ para darle forma a las estructuras ideacionales e institucionales en materia de CSS.

A lo largo de la última década, el creciente interés que la CSS ha despertado en términos políticos en los países latinoamericanos ha impulsado el intercambio de prácticas institucionales que evidencian la necesidad de darle un manejo político, pero en especial técnico. Desde esta dimensión, la CSS se desarrolla como un proceso para el intercambio de ideas y experiencias exitosas entre socios (SEGIB, 20 I I, 20I8), para lo cual, las mencionadas agencias de cooperación de los países de la región cuentan con una oficina, departamento o dirección que se encarga del manejo de la CSS.

Frente a esto último, Marsh y Sharman señalan la importancia que tienen los factores domésticos para que se lleve a cabo la difusión de políticas, los cuales incluyen las "estructuras burocráticas, el número de jugadores con poder de decisión, el ciclo de los períodos electorales" (2009, p. 279), entre otros, que influencian el intercambio y favorecen las experiencias. Los agentes adoptan reglas o instituciones que consideran apropiadas para sus entornos locales (Fehl, 20I9).

Uno de los principales aspectos para resaltar de la CSS implementada hasta el momento por parte de los países de América Latina es su búsqueda por fortalecer sus capacidades nacionales vía emulación o aprendizaje, para lo cual "el intercambio de experiencias en materia de políticas públicas y fortalecimiento institucional, formación de profesionales e incorporación de nuevas tecnologías [y prácticas] para mejorar la gestión pública” (Ayllón, 20I3, pp. 74-75) resulta fundamental.

El siguiente cuadro resume la estructura adoptada por los países de América Latina para la implementación de la CSS en la normatividad de sus agencias como un factor para el fortalecimiento de capacidades nacionales. Esto último va ligado al grado de experticia que cada país adquiere en un tema, que depende tanto de los intereses del gobierno de turno, como de la capacidad de agencia de los estados. Por ejemplo, Brasil priorizó políticas en materia de salud, educación y agroindustria; Colombia en seguridad (como herramienta para alcanzar el desarrollo) ${ }^{9}$ y bancarización, y México en cultura, educación y fortalecimiento institucional (SEGIB, 20I8).

\footnotetext{
8 Como se le conoce a la Segunda Conferencia de Alto Nivel de las Naciones Unidas sobre la Cooperación Sur-Sur.

9 Como queda reflejado en la hoja de ruta 20I5-2018 de la Agencia Presidencial de la cooperación internacional de COLOMBIA (APCColombia) en la que se destaca como tema transversal, los asuntos de paz (APC-Colombia, 20I5).
} 
Asimismo, el cuadro también incorpora las definiciones oficiales que sobre CSS han construido sus agencias, lo que demuestra la intersubjetividad de sus discursos y la posición desde la cual la entienden (política, técnica, económica). Tal como se mencionó en el primer apartado, la narrativa es un factor clave para comprender el proceso de formación, y de inserción de cada país en la arquitectura de la CID.

\section{Institucionalidad en materia de CSS de seis países de América Latina}

\begin{tabular}{|c|c|c|}
\hline Agencia & Institucionalidad CSS & Definición CSS \\
\hline $\begin{array}{c}\text { BRASIL } \\
\text { Agência Brasileira de } \\
\text { Cooperação (ABC) }\end{array}$ & $\begin{array}{l}\text { ABC cuenta con siete coordinaciones generales, } \\
\text { de las cuales dos se encargan de dirigir las acciones } \\
\text { y políticas hacia los países en desarrollo: Coorde- } \\
\text { nação-Geral de cooperação Técnica África, Ásia e } \\
\text { Oceania (CGAA),y la Coordenação-Geral de coo- } \\
\text { peração Técnica América Latina, Caribe e Europa } \\
\text { oriental (CGAE). }\end{array}$ & $\begin{array}{l}\text { "La estrategia de cooperación técnica prestada por Brasil está } \\
\text { centrada en el fortalecimiento institucional de sus socios, condi- } \\
\text { ción fundamental para que la transferencia y la absorción de los } \\
\text { conocimientos sean efectivos. Sin ánimo de lucro y desvinculada } \\
\text { de intereses comerciales, la cooperación técnica pretende com- } \\
\text { partir las mejores prácticas en las áreas demandadas por los países } \\
\text { socios" (ABC, s.f.). }\end{array}$ \\
\hline $\begin{array}{l}\text { MÉXICO } \\
\text { Agencia Mexicana } \\
\text { de Cooperación } \\
\text { Internacional para el } \\
\text { Desarrollo (Amexid) }\end{array}$ & $\begin{array}{l}\text { Amexid cuenta con cinco direcciones generales, } \\
\text { todas ellas ejecutan proyectos de CSS. Las dire- } \\
\text { cciones generales son: I. Cooperación educativa } \\
\text { y cultural, 2. Promoción económica internacional, } \\
\text { 3. Relaciones económicas bilaterales, 4. Técnica y } \\
\text { científica, 5. Proyecto de integración y desarrollo } \\
\text { de Mesoamérica. }\end{array}$ & $\begin{array}{l}\text { La CSS es "un proceso de intercambio de experiencias, conocimi- } \\
\text { ento y personal calificado entre dos o más países en desarrollo, in- } \\
\text { cluso iniciativas regionales e internacionales. Desde hace muchos } \\
\text { años, México ha estado comprometido a este tipo de intercambio } \\
\text { con países del Sur, particularmente en América Latina y el Caribe" } \\
\text { (AMEXCID, s.f.). }\end{array}$ \\
\hline $\begin{array}{l}\text { ARGENTINA } \\
\text { Dirección General } \\
\text { de Cooperación } \\
\text { Internacional del } \\
\text { Ministerio de Rela- } \\
\text { ciones Exteriores y } \\
\quad \text { Cultos }\end{array}$ & $\begin{array}{l}\text { La CSS y triangular de Argentina se impulsa a } \\
\text { través del Fondo Argentino de Cooperación Sur- } \\
\text { Sur y Triangular (FO.AR) a través del cual se busca } \\
\text { consolidar la presencia y multiplicar el impacto de } \\
\text { la cooperación argentina. }\end{array}$ & $\begin{array}{l}\text { La política de cooperación internacional de la Argentina se ha } \\
\text { construido sobre la base de nuestra pertenencia a la región la- } \\
\text { tinoamericana y al mundo en desarrollo, donde compartimos la } \\
\text { convicción de que la cooperación debe plantearse en un plano de } \\
\text { horizontalidad, en el cual los estados nos asociamos para encon- } \\
\text { trar a través del trabajo conjunto las estrategias y políticas más } \\
\text { adecuadas para responder a nuestras necesidades y superar los } \\
\text { desafíos que nos plantea el desarrollo como Estados soberanos } \\
\text { (Dirección General de Cooperación internacional, s.f.). }\end{array}$ \\
\hline $\begin{array}{l}\text { CUBA } \\
\text { Ministerio de } \\
\text { Comercio Exterior y } \\
\text { la Inversión Extran- } \\
\text { jera }\end{array}$ & $\begin{array}{l}\text { La cooperación cubana es desarrollada desde el } \\
\text { Ministerio de Comercio Exterior y la Inversión } \\
\text { Extranjera desde la Dirección General de Colabo- } \\
\text { ración Económica. Dentro de esta, Cuba también } \\
\text { tiene una dirección encargada de la cooperación } \\
\text { que ofrece y que recibe ayuda. Esta política está } \\
\text { articulada con el Ministerio de Relaciones Exte- } \\
\text { riores. }\end{array}$ & $\begin{array}{l}\text { A diferencia de los otros países, Cuba no cuenta con una defini- } \\
\text { ción específica. No obstante, se enmarca entre la visión de CTPD } \\
\text { naciente desde el PABA (1978) y que se enmarca desde un discur- } \\
\text { so político de emancipación y autonomía. }\end{array}$ \\
\hline $\begin{array}{l}\text { CHILE } \\
\text { Agencia Chilena } \\
\text { de Cooperación } \\
\text { Internacional para el } \\
\text { Desarrollo (Agcid) }\end{array}$ & $\begin{array}{l}\text { La Agcid articula su política nacional de coopera- } \\
\text { ción para el desarrollo con la demanda de los que } \\
\text { denomina países socios del Sur. La Agcid cuenta } \\
\text { con seis departamentos de los cuales dos se en- } \\
\text { cargan de coordinar y dirigir la estrategia de CSS: } \\
\text { Departamento de Formación y Becas y, Departa- } \\
\text { mento de Cooperación Horizontal. }\end{array}$ & $\begin{array}{l}\text { "Chile entiende la Cooperación Sur-Sur como una cooperación } \\
\text { técnica y no financiera, la que se realiza mediante acciones directas } \\
\text { y proyectos, e implica concentrar nuestros esfuerzos en traspa- } \\
\text { sar capacidades y conocimientos, principalmente, en materias de } \\
\text { políticas públicas y en formación de recursos humanos" (AGCl, } \\
\text { s.f.). }\end{array}$ \\
\hline $\begin{array}{l}\text { COLOMBIA } \\
\text { Agencia Presidencial } \\
\text { de Cooperación } \\
\text { Internacional de } \\
\text { Colombia (APC-Co- } \\
\text { lombia) }\end{array}$ & $\begin{array}{l}\text { APC-Colombia está organizada alrededor de } \\
\text { cuatro direcciones, desde la Dirección de Oferta } \\
\text { se coordina, gestiona y se da orientación técnica } \\
\text { alrededor de las estrategias regionales diseñadas } \\
\text { desde el Ministerio de Relaciones Exteriores. }\end{array}$ & $\begin{array}{l}\text { "Es un instrumento privilegiado de la política exterior colombiana } \\
\text { que se orienta en la generación de agendas positivas y en el } \\
\text { intercambio de experiencias y conocimientos técnicos de alto } \\
\text { valor con países en desarrollo. Diseñamos e implementamos el } \\
\text { modelo de cuantificación y agregación de valor: Metodología que } \\
\text { calcula el aporte de un proyecto de CSS en términos de recursos } \\
\text { invertidos (cuantificación) y conocimiento generado (agregación } \\
\text { de valor)" (APC-Colombia, s.f.). }\end{array}$ \\
\hline
\end{tabular}

Fuente: Elaboración propia a partir de la información oficial suministrada por cada agencia en su página web.

El análisis alrededor de la difusión de normas en materia de CSS se ha centrado en su 
carácter instrumental, en el cual, tal como se señaló, hay un proceso inicial de emulación a partir del conocimiento adquirido desde lo internacional, pero que se transforma y adapta conforme evoluciona la CID. La CSS nace como una práctica política entre los países latinoamericanos y como vía complementaria para la cooperación Norte-Sur, e incluso como contestación política a un mayor grado de autonomía (Agnew, 2003), de ahí que su práctica se instrumentalice a través de la política exterior, como evidencia algunas de las definiciones de CSS mencionadas.

\section{I. La gobernanza regional de la CSS: el caso de la SEGIB}

Durante la primera década del nuevo siglo,América Latina se caracterizó por la alineación de intereses políticos alrededor de marcos de integración regional que respondían al buen momento de la economía mundial. En esa línea, algunos autores definen este período como la década dorada de la CSS (Sotillo y Ayllón, 20I7), porque se incrementó el intercambio de proyectos entre países de la región (Xalma, 20I3).

Dentro de este contexto, la CSS tiene una gran acogida desde las organizaciones regionales, en las cuales "se consolidaron diversos espacios que contribuyeron a generar nuevos ámbitos de discusión para las autoridades en materia de cooperación” (SEGIB, 2018, p. 60), entre los más representativos: la Alianza Bolivariana para los Pueblos de Nuestra América (ALBA, 2005), la Unión de Naciones Suramericanas (UNASUR, 2008), la Comunidad de Estados Latinoamericanos y Caribeños (CELAC, 20II) y la Alianza del Pacífico (AP, 20II).

No obstante, el que mayores avances ha tenido en la definición de criterios de medición, conceptos y prácticas ha sido la SEGIB, un organismo internacional conformado por veintidós países (entre los que se encuentran Andorra, España y Portugal) que tiene entre sus funciones la de implementar y fortalecer la CSS en la región. La SEGIB ha favorecido la articulación de los intereses, ideas y políticas entre los países iberoamericanos, cuyos resultados se evidencian a través de una publicación anual sobre el estado de la CSS (SEGIB, 20I8). Dichos informes se publican desde el 2007 con el propósito de dotar a los tomadores de decisión de instrumentos técnicos y políticos, que van desde su conceptualización hasta la sistematización y registro de prácticas entre países (Ayllón, Ojeda y Surasky, 20l4).

Desde la SEGIB, la CSS se define "como una cooperación técnica basada en el fortalecimiento mutuo de capacidades, impulsada fundamentalmente a través de programas, proyectos y acciones de cooperación y ejecutada bajo las modalidades bilateral, triangular o regional" (SEGIB, 20I8, p.7I), lo cual hay que mencionar, hace parte de los consensos alcanzados a nivel iberoamericano.

Entre los datos suministrados por el informe de la SEGIB que se ha venido citando, este señala que "entre 2006 y 2015, los países de la región participaron en alrededor de 7375 programas, proyectos y acciones de CSS" (2018, p. 67), que fueron ejecutados entre todos sus miembros, pero destacándose las acciones en su rol de oferente Brasil México y Argentina, seguidos por Cuba, Chile y Colombia, razón por la cual se tomaron antes estos seis países como ejemplos para el análisis. En cuanto al rol de receptor, la mayoría de los programas, proyectos y acciones estuvieron encaminados principalmente hacia países que dentro de la región tienen una menor renta: El Salvador, Bolivia, Costa Rica, Ecuador y Guatemala. 
Entre sus principales logros puede señalarse la continua publicación de los informes, lo que demuestra los avances que la región ha venido realizando para mejorar las prácticas alrededor de la CSS, y en especial por alcanzar consensos. Por lo cual, la región es ahora un referente de buenas prácticas para otras áreas geográficas, como por ejemplo para África que, a través de la Nueva Asociación para el Desarrollo Económico de África (NEPAD), publicó en 2018 su primer informe sobre la CSS desarrollada por los países de la región, que se realizó bajo la coordinación del PNUD con el apoyo y experiencia de la SEGIB (UNDP, 20I8).

Por otro lado, los países de la región cada vez más, y en distintos escenarios, muestran “posiciones comunes sobre CSS, en diferentes escenarios internacionales" (SEGIB, 20 I8, p. 4I), lo que les ha permitido avanzar en la construcción de mecanismos de seguimiento para hacer más eficaz la CSS, así como herramientas de capacitación y construcción colectiva de conocimiento.

Sin embargo, en la búsqueda de consensos y de resultados, sus informes incluyen como producto medible de la CSS las acciones, que hacen referencia a actividades de muy corto plazo y que tienen como objeto "ejecutarse por una sola vez en modalidades o proyectos muy concretos" (Xalma, 20I3, p. 33), lo que incrementa el número de prácticas y desvirtúa su verdadero impacto.

En cuanto a esto, aún no existe consenso en sobre cómo medir la CSS. Frente a este tema hay posturas que, aunque no son tan antagónicas, no han permitido la construcción de acuerdos frente a ello. El interés por medirla, más allá de servir de insumo para conocer su verdadero impacto, responde principalmente a los intereses políticos de los gobiernos por aumentar su visibilidad internacional.

Lo anterior es un reflejo de las falencias que tiene la estructura regional frente a la definición de elementos que podrían fortalecer su agencia, y que de cierta manera le permitiera mejorar la práctica y la eficacia de la CSS. No obstante, tal como señala Prado, aunque son ejercicios incipientes, continúan "en constante construcción y perfeccionamiento, y están contribuyendo de forma gradual a mejorar la gobernanza de la CSS” (2016, p. 42).

\section{Conclusiones}

Para entender el proceso de formación de la CSS en América Latina, se analizó desde un enfoque constructivista de difusión de políticas la construcción de estructuras organizacionales, por medio de las cuales se han moldeado los intereses e ideas de los gobiernos de la región. Asimismo, se señaló cómo ha evolucionado a partir de la identificación de intereses comunes entre agentes que se perciben como iguales.

Para ello, se hizo una revisión de los hechos sociales más icónicos en la consolidación y formación de estructuras ideacionales de la CSS. Para entender lo que se comprende, define y ejecuta en la actualidad en nombre de las relaciones horizontales, se fueron abordando a lo largo del artículo, conceptos y postulados generales del enfoque constructivista, así como el de difusión.

Una vez analizado el contexto histórico surtido por los países en desarrollo para la implementación de la CTPD hasta el fortalecimiento de la CSS, se observa que su reemergencia no 
se dio sino hasta entrado el siglo XXI. La primera década del nuevo siglo fue testigo del ascenso de los países del Sur, en especial de las denominadas potencias emergentes, que ejercieron un importante rol para llevar a cabo el proceso de trasformación del imperante orden instaurado en la Guerra Fría por los países de la OCDE y los organismos internacionales a la arquitectura de la ayuda al desarrollo.

El interés de centrar el análisis en América Latina responde a dos cuestiones, por un lado, al dinamismo proyectado desde los organismos regionales para la implementación de prácticas de CSS, y por el otro, al interés de los países por encontrar diversos mecanismos de integración y concertación alrededor de problemáticas comunes en materia de desarrollo. Lo cual impulsó a la mayoría de los países en la región a implementar y fortalecer marcos normativos e institucionales para facilitar la difusión de normas, ideas y políticas, que fue analizado desde el enfoque de la difusión trasnacional de políticas. En cuanto a esto último, para Ayllón (2016), América Latina "plantea alternativas más allá de la retórica, para avanzar en un proceso no siempre fácil de reordenamiento geopolítico y geoeconómico" (p. 36).

Asimismo, se resaltó en el análisis el rol de distintos países en la región para mostrar cómo fue el proceso de adopción e implementación de políticas, normas y prácticas para el establecimiento de una estructura institucional encaminada a dinamizar su política exterior, pero también a fortalecer capacidades nacionales para mejorar sus prácticas para la difusión de políticas. En la actualidad, la CSS es un instrumento de la política exterior de los países que la practican y se ha constituido en un mecanismo que les ha dado mayor visibilidad y posicionamiento a unos estados frente a otros, en unas temáticas específicas, tal es el caso de Argentina, Brasil, Colombia o México.

Para analizar el rol de América Latina en el fortalecimiento de la gobernanza regional, el artículo analizó el rol de la SEGIB, desde cuya institucionalidad se han llevado a cabo encuentros, publicaciones y foros de discusión tendientes a la búsqueda de consensos, así como la identificación de prácticas comunes en materia de CSS. En este sentido, la SEGIB como organización internacional ha servido de vehículo para la difusión política de prácticas e ideas entre los países de la región a partir de la identificación de problemáticas e intereses comunes.

Sin desconocer el rol que los organismos regionales o que los propios países han desempeñado en el desarrollo y gestión de la CSS, este artículo se centró en analizar cómo a lo largo de la última década los países de la región han venido trabajando en el marco institucional creado por la SEGIB para dinamizar la CSS, un ejercicio que va desde visibilizar las prácticas de la CSS de todos los países de la región, hasta la sistematización de procesos técnicos que han servido de insumo para la definición de intereses políticos. Porque finalmente, y tal como lo señala Ayllón (2016), “la posibilidad de construir la gobernanza global del desarrollo y la cooperación desde los espacios, foros y mecanismos de integración, cooperación y diálogo político en América Latina es una alternativa creíble y factible" (p. 39) y en este proceso, la SEGIB ha favorecido la transferencia de normas, prácticas y políticas, que para el caso estudiado ha girado alrededor de fortalecer la CSS latinoamericana. 


\section{Referencias}

Agência Brasileira de Cooperação (ABC) (s.f). Histórico. Recuperado de: http://www.abc.gov.br (I2.04.2019).

Agencia Chilena de Cooperación Internacional al Desarrollo (AGCI) (s.f). Nuestra historia. Recuperado de: https:// www.agci.cl (12.04.2019).

Agencia Mexicana de Cooperación Internacional para el Desarrollo (AMEXCID) (s.f.). Qué hacemos. Recuperado de: https://www.gob.mx/amexcid (I 2.04.20I9).

Agencia Presidencial de Cooperación Internacional de Colombia (APC-Colombia) (20I5). APC-Colombia lanza la Hoja de Ruta de la Cooperación Internacional 2015-2018. Recuperado de: https://www.apccolombia.gov.co/ node/I59 (I 2.04.20I9)

Agencia Presidencial de Cooperación Internacional de Colombia (APC-Colombia) (s.f.). SobreApc-Colombia, cooperación Sur-Sur. Recuperado de: https://www.apccolombia.gov.co/sur-sur (I 2.04.20 I9).

Agencia Presidencial de Cooperación Internacional de Colombia (APC-Colombia) (20I7). Manual: caja de herramientas de la Cooperación Sur-Sur. En APC-Colombia, Cooperación Sur-Sur. Recuperado de: https://www.apccolombia.gov.co/sites/default/files/manualcss-2019.pdf (I2.04.2019).

Agencia Presidencial de Cooperación Internacional de Colombia (APC-Colombia) (2019). La cuantificación y valorización de la Cooperación Sur-Sur: Retos y desafios, en Segunda Conferencia de Naciones Unidas para la cooperación Sur-Sur, Buenos Aires.

Agnew, J. (2003). Geopolitics, re-visioning world politics. New York: Routledge.

Antonini, B. y Hirst, M. (2009). Pasado y presente de la cooperación norte-sur para el desarrollo. Working Paper, Buenos Aires, pp. I-I43. Recuperado de: https://airamvl.files.wordpress.com/2015/03/cooperacion_sur_sur.pdf $(19.05 .2016)$

Ayllón, B. (2007). La Cooperación Internacional para el Desarrollo: fundamentos y justificaciones en la perspectiva de la Teoría de las Relaciones Internacionales. Carta Internacional, 2(2), 32-47. Recuperado de: https://www. cartainternacional.abri.org.br/Carta/article/view/4I6 (20.05.2015).

Ayllón, B. (20I3). La Cooperación Sur-Sur y Triangular. Quito: iaen.

Ayllón, B., Ojeda, T. y Surasky, J. (Coord.) (20l4). Cooperación Sur-Sur: Regionalismos e integración en América Latina. Madrid: Catarata.

Ayllón, B. (2016). La dimensión regional de la Cooperación Sur-Sur:América Latina y la gobernanza de la cooperación internacional. En De Souza, M. y Soares, O (Coords.) Cooperação Sul-Sul: práticas, abordagens e desafios (pp. 2I-55). Belo Horizonte: Fino Traço.

Ayllón, B. (2018). CSS y Triangular en los escenarios globales y regionales 201 2-20I6. Recuperado de: https://www.oecd. org/dac/dac-global-relations/20 I820SEGIB\%20-\%20La\%20CSS\%20y\%20TC\%20en\%20global\%20and\%20 regional\%20scenarios.pdf

Bergamaschi, I. y Soulé-Kohndou, F. (20I6). Gobernanza mundial de la ayuda al desarrollo: Estrategias reformadoras, rivalidades y tentativas. Revista Foro Internacional, LVI (I), 82-I 19. Recuperado de: http://www.redalyc.org/ articulo.oa?id=5994555 I004\%0A (06.04.2019).

Bergamashi, I. y Tickner, A. (20I7). Introduction: South-South Cooperation Beyond the Myths. A critical analysis. En Bergamaschi, I., Moore, P. y Tickner, A. (Eds.). South-South Cooperation beyond the Myths. Rising donors, New Aid Practices? (pp. I-27). London: Palgrave.

Burchill, S. (2005). The National interest in International Relations. London: Palgrave macmillan.

Chaturvedi, S., Fues, T. y Sidiropoulos, E. (Eds.) (20I2). Development Cooperation and Emerging Powers. London: Zed Books.

Checkel, J. (2008). Constructivism and foreign policy. En Smith, S., Hadfield, A. y Dunne, T. (Eds.) Foreign policy: theories, actors, cases (pp. 72-82). New York: Oxford University Press.

Commission on Global Governance (1995). Our Global Neighborhood.The Report of the Commission on Global Governance. Oxford: Oxford University Press.

Dirección General de Cooperación Internacional (s.f.) Cooperación Técnica Internacional Argentina en el mundo. Recuperado de: http://cooperacionarg.gob.ar (12.04.2019).

Dolowitz, D. y Marsh, D. (2000). Learning from Abroad:The role of Policy Transfer in Contemporary Policy-Making. Governance:An International Journal of Policy and Administration, I 3 (I), 5-24. DOI: I0. I I I I/0952- I895.00 I 2 I

Engel, P.y Keijzer, N. (20I3). Development Policy on the Edge:Towards a post-20 I 5 global, I4I, pp. I - I3. Maastricht: ecdpm. Recuperado de: https://www.die-gdi.de/uploads/media/DP_I4I_-_Development_Policy_on_the_Edge.pdf

Finnemore, M. y Katheryn, S. (1998). International norm dynamics and political change. International Organization, 52 (4), 887-9I7. Recuperado de: www.jstor.org/stable/260I36I (20.05.20I5).

Finnemore, M. y Sikkink, K. (200I). Taking Stock:The Constructivist Research Program in International Relations and Comparative Politics. Annual Review of Political Science, (4), 391-416. Recuperado de: https://www.annualreviews.org/doi/full/I0.I I46/annurev.polisci.4.I.39I (06.04.20I9).

Fehl, C. Navigating Norm Complexity: A Shared Research Agenda for Diverse Constructivist Perspectives, Peace Research Institute Frankfurt, Working Paper, $\mathrm{n}^{\circ}$ 4I/20 I8, pp. I- I5. Recuperado de: https://www.ssoar.info/ssoar/handle/ document/59468 (23.05.2019)

Fraundorfer, M. (20I5). Brazil's Emerging Role in Global Governance: Health, Food Security and Bionergy. London: Palgrave macmillan

Gilardi, F. (20I3). Transnational Diffusion: Norms, Ideas, and Policies. En Carlsnaes, W., RISSE, T. y Simmons, B. (Eds.), 
Handbook of International Relations (pp. 453-477). London: SAGE.

Hulme, D. (2009). The Millennium Development Goals (MDGs):A Short History of the World's Biggest Promise. Recuperado de: https://papers.ssrn.com/sol3/papers.cfm?abstract id=|54427|

Ibáñez, J. (20I5). Socialconstructivismo: ideas, valores y normas en la política mundial. En Del Arenal, C.y Sanahuja, J.A. (Coords.) Teorías de las Relaciones Internacionales (pp. 189-217). Madrid:Tecnos.

Jiménez, C. (2003). Las teorías de la cooperación internacional dentro de las relaciones internacionales. Polis: Investigación y Análisis Sociopolítico y Psicosocial, 3 (2), I I5-I47. Recuperado de: http://www.redalyc.org/articulo. oa?id=72620305 (20.05.2015).

Karns, M., Mingst, K., Stiles, K. (20I5). International Organizations:The Politics and Processes of Global Governance. Boulder, C: Lynne Rienner.

March, J., y Olsen, J. (1998). The Institutional Dynamics of International Political Orders. International Organization, 52 (4), 943-969. Recuperado de: www.jstor.org/stable/260I 363 (I2.04.20I9).

Marsh, D. y Sharman, J. (2009). Policy diffusion and policy transfer. Policy Studies, 3 (30), 269-288. DOI: 10.1080/0|442870902863385|

Mawdsley, E. (2012). The changing geographies of foreign aid and development cooperation: Contributions from gift theory. Transactions of the Institute of British Geographers, (37), 256-272.DOI: I 0. I I I I/j. I 475-566 I.20 I I.00467.x

Mawdsley, E. (2015). Development geography I: Cooperation, competition and convergence between 'North' and 'South.' Progress in Human Geography, I (4I), I08-I I7. DOI: I0.I I 77/0309I325I560I776

Mawdsley, E. (2018). The 'Southernisation' of development?. Asia Pacific Viewpoint, 2 (59), I73-I85. DOI: I0.IIII/ apv. 12192

Milani, C. (20I8). Solidariedade e interesse. Motivações e estratégias na cooperação internacional para o desenvolvimento. Curitiba:Appris.

Naciones Unidas (04.12.1974). Cooperación Técnica entre los Países en Desarrollo. Recuperado de:

https://www.un.org/ga/search/view doc.asp?symbol=A/RES/325I(XXIX)\&referer=/english/\&Lang=S (07.05.20I6).

Naciones Unidas (2000). Declaración del Milenio. Recuperado de: https://www.un.org/spanish/milenio/ares552.pdf

Naciones Unidas (2010). Documento final de Nairobi. Recuperado de: https://undocs.org/pdf?symbol=es/A/RES/64/222 (07.05.2016).

Ojeda,T. (2016). Relaciones Internacionales y cooperación con enfoque Sur-Sur. Madrid: Catarata.

Onuf, N. (1998). Constructivism: A User's Manual. En Kubálková,V., Onuf, N. y Kowert, P. (Eds.). International Relations in a Constructed World (pp. 58-78). New York: Routledge.

Pauselli, G. (20/3). Teorías de relaciones internacionales y la explicación de la ayuda externa. Revista lberoamericana de Estudios de Desarrollo, I (2), 72-92. Recuperado de: http://ried.unizar.es/index.php/revista/article/viewFile/65/29 (I5.08.20I5)

Prado, J. P. (20I6). La gobernanza de la Cooperación Sur-Sur. Condicionantes, logros y desafíos desde una perspectiva latinoamericana. En Soares, M. R. y Milani, C. (Eds.). Política externa, geopolítica e modelos de desenvolvimento (pp. 4I-65). Buenos Aires: Clacso.

Quadir, F. (20I3). Rising donors and the new narrative of "South-South" cooperation: What prospects for changing the landscape of development assistance programmes?. Third World Quarterly, 34 (2), pp. 32I-338. DOI: $10.1080 / 01436597.2013 .775788$

Ruggie, J. (1998). What Makes the World Hang Together? Neo-utilitarianism and the Social Constructivist Challenge. International Organizations, 52 (4), 855-885. Recuperado de:

http://links.jstor.org/sici?sici=0020-8I83\%28I 99823\%2952\%3A4\%3C855\%3AWMTWHT\%3E2.0.CO\%3B2-8

Ruiz, P. (2018). La agenda de la cooperación internacional al desarrollo en Colombia (2010- 20I8). En Soto, C. (Ed.). Seguimiento y análisis de políticas públicas en Colombia (I73-191). Bogotá: Universidad Externado de Colombia. Recuperado de: https://publicaciones.uexternado.edu.co/pageflip/acceso-abierto/pdf/seguimiento-y- analisis-de-politicas-publicas-en-colombia-uext.pdf

Sanahuja, J.A. y Tezanos, S. (2016). Del milenio a la sostenibilidad: Retos y perspectivas de la Agenda 2030 para el desarrollo sostenible. Política y Sociedad, 2 (54) 52I-543. DOI:I0.5209/POSO.5I 926

Santander, G. (2016). Identidades e intereses en la cooperación Sur-Sur, Madrid: Catarata.

Secretaria General lberoamericana (SEGIB) (20II). Informe de la cooperación Sur-Sur en lberoamérica 20II. Recuperado de https://www.SEGIB.org/wp-content/uploads/Informe-Sur-Sur-20 I I-ESP.pdf (I2.04.20I9).

Secretaria General Iberoamericana (SEGIB) (20I8). Una década de Cooperación Sur-Sur en Iberoamérica. Madrid:AECID.

Sikkink, K. ( I99I). Ideas and Institutions. Developmentalism in Brazil and Argentina. Ithaca: Press, Cornell University.

Sotillo, J. Á. y Ayllon, B. (20 I7). Las transformaciones de América Latina. Cambios políticos, socioeconómicos y protagonismo internacional. Madrid: Catarata.

Sousa, S.L. (20I3). Cambios en el poder estructural y países emergentes. Brasilia: Coleção política externa brasileira. Recuperado de: http://funag.gov.br/loja/download/I029-Cambios_en_el_Poder_Estructural_y_Palses_Emeregentes.pdf (12.04.2019)

Surasky,J. (20I5). Cooperación Sur-Sur en la Agenda 2030 y hacia el Paba+40,Working Paper. Recuperado de: http://www. iri.edu.ar/wp-content/uploads/2017/09/depto_cooperacion_surasky_sur_sur_paba40.pdf (I0.04.2018).

Surasky, J. y Ayllón, B. (20I8). Desarrollo y cooperación. Revista Española de Desarrollo y Cooperación, (43), 37-5I.

United Nations Development Program (UNDP) (1978). The Buenos Aires Plan of Action. Recuperado de: https://drive. google.com/file/d/OB-buqyoV0jpSMm IOVEZYU2hNTWc/view (I5.08.20I5). 
United Nations Development Program (UNDP) (12.06.2018). South-South Cooperation in Sub-Saharan Africa: Strategies for UNDP engagement. Recuperado de: https://www.africa.undp.org/content/rba/en/home/library/reports/ south-south-cooperation-in-sub-saharan-africa0.html (I5.II.2019).

United Nations Office for South-South Cooperation (UNOSSC) (2018). Acerca de la UNOSSC. Recuperado de: https://www.unsouthsouth.org/acerca-de/acerca-de-la-unossc/?lang=es

Viola, C. (20I4). Diagnóstico de los marcos normativos e institucionales para la gestión de la Cooperación Sur-Sur en los países de Iberoamérica. Recuperado de: https://www.SEGIB.org/?document=diagnostico-de-los-marcos-normativos-e-institucionales-para-la-gestion-de-la-css-en-ios-paises-de-iberoamerica-documento-de-trabajo-no-6ano-20I 4 (06.04.2019).

Wendt,A. ( I 987). The Agent-Structure Problem in International Relations Theory. International Organization, 3 (4I), pp. 335-370. DOI: 10.10I7/S00208183000275IX

Wendt, A. (1992). Anarchy is what States Make of it:The Social Construction of Power Politics. International Organization, 2 (46), 39l-425. Recuperado de: www.jstor.org/stable/2706858

Wendt, A. (1995). Constructing International Politics. International Security, I (20), 7I-8I. Recuperado de: http://www. jstor.org/stable/2539217

Wight, C. (2006). Agents, Structures and International Relations. Politics and Ontology. Cambridge: Cambridge University Press.

Xalma, C. (20I3). El renovado auge de la Cooperación Sur-Sur: La experiencia iberoamericana. Obtenido de Instituto para la integración de América Latina y el Caribe. Recuperado de: http://www20.iadb.org/intal/catalogo/integracion_comercio/e_INTAL_IYC_36_20I3_Xalma.pdf (19.05.2016). 
Relaciones Internacionales

Número 44 • Junio 2020 - Septiembre 2020

Grupo de Estudios de Relaciones Internacionales

Universidad Autónoma de Madrid 


\section{RELACIONES INTERNACIONALES}

Revista académica cuatrimestral de publicación electrónica Grupo de Estudios de Relaciones Internacionales (GERI)

Universidad Autónoma de Madrid, España

https://revistas.uam.es/relacionesinternacionales

ISSN 1699 - 3950

f facebook.com/RelacionesInternacionales

3. twitter.com/RRInternacional

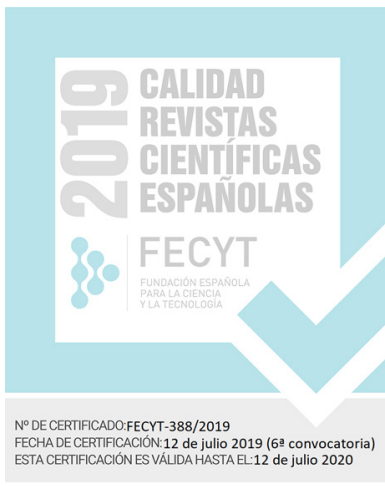

